\title{
A205 REDUCED LEVELS OF THE TGFB FAMILY MEMBER GDF15 IN SPONDYLOARTHRITIS VERSUS OTHER RHEUMATIC DISEASES
}

Lambrecht S, Coudenys J, De Keyser F, Verbruggen G, Deforce D, Elewaut D Department of Rheumatology, Ghent University, Ghent

10.1136/ard.2010.149021.15

Introduction The transforming growth factor (TGF)- $\beta$ superfamily consists of a number of molecules that regulate a variety of cellular processes such as growth, differentiation and oncogenesis. Growth differentiation factor 15 is a distant member of this TGF- $\beta$ family. GDF15 was previously shown to be elevated in serum of rheumatoid arthritis (RA)-patients compared to healthy controls. This study aims to compare GDF15 serum and synovial fluid levels in several inflammatory rheumatic diseases.

Methods GDF15 levels were determined by ELISA in two different cohorts. A first group included serum samples from patients with an indication for an arthroscopic procedure for diagnostic purposes. A total of 37 RA patients, 63 spondyloarthritis (SpA) patients and 17 osteoarthritis patients was analysed. Synovial fluid levels in RA and SpA patients from this cohort were determined as well. A second confirmatory cohort constituted of a consecutive cohort of 555 patients visiting the outpatient clinic of the department of Rheumatology at Ghent University Hospital.

Results SpA samples show a significant lower serum concentration of GDF15 compared to RA patients. When SpA patients were stratified according to the subdiagnosis (USpA, AS or PsA) no statistically significant differences could be observed between the groups. Interestingly, SpA patients, but not RA-patients, show a significant higher concentration of GDF15 in the synovial fluid compared to serum (serum $=516.38 \mathrm{pg} / \mathrm{ml} \pm 71.09$ vs syn fluid $803.2167 \mathrm{pg} / \mathrm{ml} \pm 99.14$; paired sample t-test, $\mathrm{p}<0.001$ ), pointing to a local production of GDF15 in the synovial joint. No significant correlations were observed between GDF15 concentration and routine biochemical (C-reactive protein, erythrocyte sedimentation rate) or clinical markers (number of swollen joints, DAS28), indicating that GDF15 serum levels might be indicative for a distinct underlying disease process.

Analysis the second group consisting of a consecutive cohort of 555 patients confirmed the lower concentration of GDF15 in serum samples of SpA patients compared to RA patients. To estimate the diagnostic potential to discriminate SpA from RA patients, a ROC curve analysis was performed, characterised by a AUC of 0.76 . In addition, it was demonstrated that GDF15 levels might have an added value to anti-CCP and rheumatoid factor to discriminate RA and SpA patients.

Conclusion GDF15 serum concentrations are significantly lower in SpA patients compared to other inflammatory joint diseases. The serum levels are not directly correlated to inflammatory or known diagnostic parameters and thus may serve as an additional marker for diagnostic purposes in inflammatory joint diseases. 